\title{
Gastrodin ameliorates Parkinson's disease by downregulating connexin 43
}

\author{
YU WANG, ZHE WU, XU LIU and QUNYING FU \\ Department of Neurology, The First Hospital of China Medical University, Shenyang, Liaoning 110001, P.R. China
}

Received March 4, 2013; Accepted June 13, 2013

DOI: $10.3892 / \mathrm{mmr} .2013 .1535$

\begin{abstract}
Gastrodin, the predominant constituent of a Chinese herbal medicine, has been utilized in the prevention of Parkinson's disease (PD); however, its mechanism of action remains unknown. Astrocytes are involved in PD and are proposed to be coupled with gap junction connexin 43 (Cx43). To evaluate the effects of gastrodin on PD, the effect of gastrodin on Cx43 in astrocytes and in a PD model were observed. Different doses of gastrodin were added to the astrocyte culture medium or injected into the rotenone model of PD. The relative expression of Cx43 was determined by qPCR and western blot analysis, while gap junctional intercellular communication (GJIC) was quantified using fluorescence recovery after photobleaching (FRAP). The phosphorylated Cx43 was significantly inhibited by gastrodin and the quantity of GJIC was significantly downregulated compared with that of the control cells $(\mathrm{P}<0.05)$. In addition, in the rat model of $\mathrm{PD}$ induced by rotenone, phosphorylated Cx43 was selectively enhanced in the striatal and hippocampal regions. The enhanced activity was inhibited significantly by gastrodin treatment $(\mathrm{P}<0.01)$. Gastrodin results in the prevention of $\mathrm{PD}$ by reducing the expression of $\mathrm{Cx} 43$ and inhibiting the phosphorylation of $\mathrm{Cx} 43$; therefore, it may offer a potential therapeutic alternative for patients with PD.
\end{abstract}

\section{Introduction}

Parkinson's disease (PD), a progressive movement disorder, is one of the most common neurodegenerative disorders worldwide (1). The predominant pathological features of PD are a loss of the dopaminergic (DA) neurons in the substantia nigra and striatum (2-5). Thus, stem cells may offer an alternative source of novel cells for patients with PD. It is hypothesized that the introduction of stem cells into the brain may delay

Correspondence to: Dr Zhe Wu, Department of Neurology, The First Hospital of China Medical University, 33 Wenyi Rd, Shenhe, Shenyang, Liaoning 110001, P.R. China

E-mail: zhewusy@163.com

Key words: gap junctional intercellular communication, connexin 43, Parkinson's disease model, gastrodin, rotenone the onset or progression of PD (6). However, the quantity of fetal tissue available is insufficient to treat the large number of patients with PD, and the use of neurons from fetal sources raises ethical questions. Currently, iPS cells produced from the cells of patients with Parkinson's disease are being utilized to produce diseased neurons in the laboratory, in order to determine the mechanisms of PD and to test potential therapeutic agents (7). However, whether agents with therapeutic potential in the PD models would be beneficial in patients with PD has not yet been elucidated. Furthermore, with regard to the transplantation of healthy cells into the brains of patients with PD, further studies are required to ensure the cells are safe. In addition, further investigation is required to improve the effectiveness of the transplants, minimize the side-effects, determine the mechanism of the disease and demonstrate how the cells may aid in the development of novel therapeutic agents.

Previously, cancer studies have focused on traditional medicinal plants to discover novel therapeutic agents with minimal side-effects. The use of medicinal herbs has a long history in Asia and is commonly utilized in the treatment of various neurological diseases, including stroke and epilepsy (8-10). According to ancient Chinese medical literature, Tianma (Gastrodia elata Blume, Orchidaceae) is a herbal medicine for the the treatment of PD. The dry tuber of Tianma is officially listed in the Chinese Pharmacopoeia and is utilized in the treatment of headaches, dizziness, tetanus, epilepsy, infantile convulsions and numbness of the limbs (11). Recently, gastrodin, the predominant and bioactive component of Tianma, has been demonstrated to inhibit neuroinflammation in a PD model in rats (12).

A mitochondrial complex I inhibitor, rotenone, led to the selective death of DA neurons and Parkinsonism in rodents $(13,14)$. This PD model is superior for use in the present study on the effects of gastrodin on PD. Accumulating data have indicated the importance of astrocytes in Parkinsonism (15-17). It has also been demonstrated that several connexins are expressed in neurons and astrocytes, and these may be involved in the release of ATP and glutamate (18-21). In addition, astrocytes have been shown to be involved in neurological disorders, including PD $(15,16)$, and astrocyte gap junctions may be formed of multiple connexins (22). The metabolic and ionic coupling provided by these diverse types of gap junctions may provide intercellular signaling required for brain development and cortical lamina- 
tion (19). Furthermore, astrocytes in PD are demonstrated to upregulate the expression of gap junction connexin 43 (Cx43) genes (23).

Gastrodin may inhibit $\mathrm{Cx} 43$ expression in the temporal lobe and hippocampus, inhibit the formation of abnormal gap junctions and achieve anti-epileptic formation with the suppression of aberrant new cell formation (24). The aim of the present study was to determine whether gastrodin prevents PD via its effect on the expression of $\mathrm{Cx} 43$. Thus, following gastrodin treatment, the changes in astrocyte gap junctional intercellular communication (GJIC) and Cx43, and the phosphorylation status of Cx43 were determined in a rat model of PD (induced by chronic exposure to rotenone) and in cultured astrocytes stimulated with rotenone. This model has been previously utilized to investigate the etiology of Parkinsonism $(13,25,26)$ and will aid in the study of the function of gastrodin in treating PD.

\section{Materials and methods}

Drugs and chemicals. Gastrodin injections were purchased from Nanchong Central Hospital (Nanchong, China). Rotenone and dimethylsulfoxide (DMSO) were purchased from Sigma-Aldrich (St. Louis, MO, USA). Rotenone was dissolved in DMSO and stored at $-20^{\circ} \mathrm{C}$.

Lewis rats. Lewis rats (weight, 200-250 g) were purchased from the Shanghai Laboratory Animal Centre (Chinese Academy of Sciences, Shanghai, China) and maintained in specific pathogen-free conditions. The rats were acclimated and maintained at $23^{\circ} \mathrm{C}$ under a 12-h light/dark cycle (lights on, 08:00-20:00). Rats were housed in standard laboratory cages with free access to food and water. The rats were randomly divided into experimental $(n=6)$ and control $(n=12)$ groups. The experimental group subcutaneously received rotenone and gastrodin (2.5 and $5.0 \mathrm{mg} / \mathrm{kg}$, respectively, in Panacet) and the control group received Panacet only. All procedures were conducted in accordance with the National Institutes of Health Guide for the Care and Use of Laboratory Animals (27) and were approved by the animal care committee of China Medical University (Shenyang, China).

Primary astrocyte cultures. Primary astrocytes were prepared from the brains of neonatal Wistar rats (age, 1-2 days) (28), which were purchased from Shanghai SLAC Laboratory Animal Co. (Shanghai, China). Briefly, the brains were digested with $0.05 \%$ trypsin-EDTA at $37^{\circ} \mathrm{C}$ for $10 \mathrm{~min}$, dissociated by gentle pipetting and passed through a $100-\mu \mathrm{m}$-pore nylon mesh. Cells were plated onto $75-\mathrm{cm}^{2}$ plastic flasks and grown in Dulbecco's Modified Eagle's Medium (DMEM) supplemented with 10\% $\mathrm{v} / \mathrm{v}$ fetal bovine serum and $1 \%$ penicillin/streptomycin, at $37^{\circ} \mathrm{C}$ in a humidified $5 \% \mathrm{CO}_{2}$ atmosphere. The medium was changed every three days. Cells were harvested when they reached $80 \%$ confluence and seeded into a secondary culture. The purity of the primary astrocyte cultures was determined by immunocytochemical staining using an antibody against an astrocyte-specific marker, glial fibrillary acidic protein (GFAP; dilution, 1:1000; product number G 3893, Sigma) or a microglia-specific marker (anti-CD11b; dilution, 1:200; Serotec, Oxford, UK). At 30 days in vitro, $99 \%$ of the primary cultured cells were GFAP-positive and no detectable CD11b- positive cells (microglia) were identified (29). Cultured astrocytes were treated with rotenone $(8 \mathrm{nM})$ and gastrodin (10 or $20 \mathrm{nM}$; molecular formula, $\mathrm{C}_{13} \mathrm{H}_{18} \mathrm{O}_{7}$; molecular weight, 286.25), or with $8 \mathrm{nM}$ rotenone only for 2 days.

Fluorescence recovery after photobleaching (FRAP) assay for GJIC. The quantitative FRAP assay for GJIC was performed as previously described (23), using a laser-scanning confocal microscope (LSCM, Olympus Fluoview FV300; Olympus, Ltd., Beijing, China). Following the bleaching of randomly selected cells with a micro-laser beam, the rate of transfer of 5,6-carboxyfluoresceindiacetate (Sigma-Aldrich) from adjacent labeled cells back into the bleached cells was calculated. The recovery of fluorescence was examined after $0.5 \mathrm{~min}$ and the recovery rate (RR) was calculated as the percentage of photobleached fluorescence/min. The RR was adjusted for the loss of fluorescence measured in unbleached cells and the results are expressed as the fold increase in the RR compared with that of the untreated control cells (23).

Extraction of Cx43 RNA and the quantification of Cx43 mRNA. Cells were grown in 6 -cm cell culture dishes for $\geq 48 \mathrm{~h}$. The cells were trypsinized and suspended in DMEM containing $10 \%$ fetal calf serum. Total RNA was isolated from the cells using the QIAshredder and RNeasy mini kits (Qiagen, Inc., Almeda, CA, USA). The initial strand of cDNA was synthesized from $500 \mathrm{ng}$ RNA extracts in a volume of $20 \mu \mathrm{l}$ using AMV reverse transcriptase XL (Takara Biotechnology Co., Ltd., Dalian, China) priming with random nonamer primers (9-mers) at $42^{\circ} \mathrm{C}$ for $10 \mathrm{~min}$. The cDNA strand was stored at $20^{\circ} \mathrm{C}$ until use. The expression of $\mathrm{Cx} 43$ mRNA was determined by qPCR. PCR was performed in an ABI Prism 7900 sequence detector (Applied Biosystems, Foster City, CA, USA) in a final volume of $20 \mu \mathrm{l}$. The PCR mixture contained $10 \mathrm{mM}$ Tris- $\mathrm{HCl}$ buffer (pH 8.3), $50 \mathrm{mM} \mathrm{KCl}, 1.5 \mathrm{mM} \mathrm{MgCl}_{2}$, $0.2 \mathrm{mM}$ dNTP mixture, 0.5 units Ampli Taq gold enzyme (Applied Biosystems) and 0.2 M primers. The primer and probe sequences for gene amplification were as follows: $\mathrm{Cx} 43$ forward, 5'-ATCAGCATCCTCTTCAAGTCTGTCT-3' and reverse, 5'-CAGGGATCTCTCTTGCAGGTGTA-3' (22); and glyceraldehyde 3-phosphate dehydrogenase (GAPDH) forward, 5'-CCCTTCATTGACCTCAACTAC-3' and reverse, 5'-CCACCTTCTTGATGTCATCAT-3'. GAPDH was used as an internal control. The Ampli Taq gold enzyme was activated by heating for $10 \mathrm{~min}$ at $95^{\circ} \mathrm{C}$, and all genes were amplified by 50 cycles of heating for $15 \mathrm{sec}$ at $95^{\circ} \mathrm{C}$, followed by $1 \mathrm{~min}$ at $60^{\circ} \mathrm{C}$.

For the construction of standard curves for the positive controls, the total RNA of the primary astrocytes was reverse transcribed into cDNA and serially diluted in water in five or six log steps to achieve four-fold serial dilutions of cDNA from $\sim 100 \mathrm{ng}$ to $100 \mathrm{pg}$. These cDNA serial dilutions were stored at $-20^{\circ} \mathrm{C}$. The coefficient of linear regression for each standard curve was calculated, and the cycle threshold value of a sample was substituted into the formula for each standard curve to calculate the relative concentration of $\mathrm{Cx} 43$ or GAPDH. To normalize the differences in the quantity of total RNA added to each reaction mixture, GAPDH was used as an endogenous control. The data represent the average expression of target genes relative to GAPDH, from three independent cultures. 

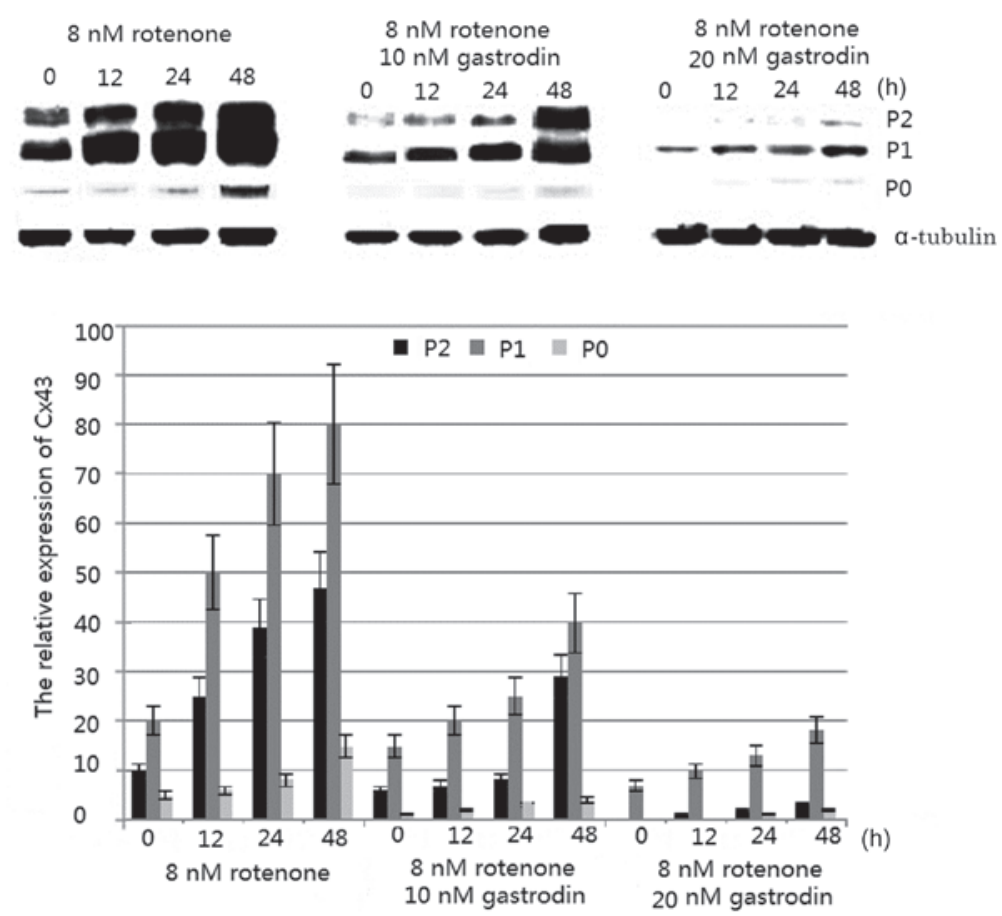

Figure 1. Gastrodin reduces the protein levels of phosphorylated $\mathrm{Cx} 43$, as analyzed by western blotting. Astrocytes were cultured with rotenone ( $8 \mathrm{nM})$ and gastrodin $(10$ or $20 \mathrm{nM})$ for $0-48 \mathrm{~h}$. $\alpha$-tubulin was used as an internal control. The relative expression of Cx43 was calculated as relative to the value of CX43 expression in untreated astrocytes. Data are presented as the mean $\pm \mathrm{SE}(\mathrm{n}=3)$. P0, non-phosphorylated form of $\mathrm{Cx} 43$; P1 and P2, phosphorylated forms of Cx43.

Western blot analysis. Cells and rat brains were lysed in icecold buffer $(50 \mathrm{mmol} / \mathrm{l}$ Tris- $\mathrm{HCl}, \mathrm{pH} 7.4 ; 150 \mathrm{mmol} / \mathrm{l} \mathrm{NaCl}$; $1 \%[\mathrm{v} / \mathrm{v}] \mathrm{NP} 40 ; 5 \mathrm{mmol} / 1$ EDTA; $5 \%$ [v/v] glycerol; $10 \mu \mathrm{g} / \mathrm{ml}$ leupeptin; $10 \mu \mathrm{g} / \mathrm{ml}$ aprotonin; $1 \mathrm{mmol} / 1$ phenylmethylsulfonyl fluoride and $1 \mathrm{mmol} / 1 \mathrm{Na}_{3} \mathrm{VO}_{4}$ ) using a polytron. The lysates were then sonicated, the samples were diluted 1:4 in water and their protein concentrations were determined using the Bradford method (30), with affinity-purified bovine serum albumin as a standard. Samples $(10 \mathrm{~g})$ were dissolved in Laemmli sample buffer (60 mM Tris- $\mathrm{Cl}$ pH 6.8, 2\% SDS, $10 \%$ glycerol, $5 \% \beta$-mercaptoethanol, $0.01 \%$ bromophenol blue), separated on $12 \%$ acrylamide gel and transferred to polyvinylidene fluoride (PVDF) membranes. Blots were incubated with anti-Cx43 antibody (Shengshizhongfang BioSci and Tech. Co., Ltd., Beijing, China) overnight at $4^{\circ} \mathrm{C}$, followed by three $15 \mathrm{~min}$ washes with phosphate-buffered saline and $0.1 \%$ Triton X-100 (PBST). As an internal control, to determine whether equal amounts of protein had been loaded onto the gel, the PVDF membranes were stripped and reprobed with anti-tubulin (T5168; Sigma-Aldrich). Blots were incubated with goat anti-rabbit antibody conjugated horseradish peroxidase (AP307P, Merck Millipore, Billerica, MA USA). The immunoreactive bands were visualized by enhanced chemiluminescence (ECL; GE Healthcare, Shanghai, China) and quantified by densitometry with ImageJ 1.45 software (National Institutes of Health, Bethesda, USA) according to the manufacturer's instructions.

Statistical analysis. The correlation between Cx43 levels and gastrodin and rotenone treatment in the different groups was compared by a one-way analysis of variance followed by post hoc analysis with a protected Fisher's least significant difference test. $\mathrm{P}<0.05$ indicates a statistically significant difference.

\section{Results}

Gastrodin inhibits the rotenone-induced levels of Cx43 expression in astrocytes. Western blot analysis demonstrated that three forms of the Cx43 immunoreactive protein ( $\mathrm{Mr} 40,000-43,000)$ were observed in all samples; a fast-migrating band (non-phosphorylated form, P0; Fig. 1) and two slower migrating bands (phosphorylated forms, P1 and P2; Fig. 1). Phosphorylated Cx43 was observed to localize at the plasma membrane and gap junctions (23). Densitometric analysis demonstrated that rotenone induced a significant dose- and time-dependent increase in phosphorylated Cx43 levels compared with that of the control cells (23). The levels of the non-phosphorylated form, P0, appeared to marginally change (Fig. 1). The effect of rotenone on Cx43 protein levels was also determined, and the phosphorylated Cx43 level was demonstrated to be modulated by rotenone treatment. The expression of phosphorylated $\mathrm{Cx} 43$ reached high levels when the astrocytes were treated with $8 \mathrm{~nm}$ rotenone for $48 \mathrm{~h}$ (Fig. 1). However, the enhanced expression level of phosphorylated Cx43 was inhibited by gastrodin. The increased inhibitory rate was correlated with an increasing concentration of gastrodin, and was greatest when $20 \mathrm{nM}$ gastrodin was added (Fig. 1).

Quantification analysis also demonstrated that gastrodin inhibited the rotenone-induced levels of $\mathrm{Cx} 43$ expression in astrocytes. The effect of rotenone on Cx43 mRNA levels was also investigated by qPCR. Rotenone treatment was observed to modulate the $\mathrm{Cx} 43 \mathrm{mRNA}$ levels. Following the treatment of astrocytes with $8 \mathrm{~nm}$ rotenone for $48 \mathrm{~h}$, the Cx43 mRNA levels increased (Fig. 2). The enhanced mRNA levels of Cx43 were shown to be inhibited by gastrodin. In addition, the increased inhibitory rate was correlated with increasing concentrations 


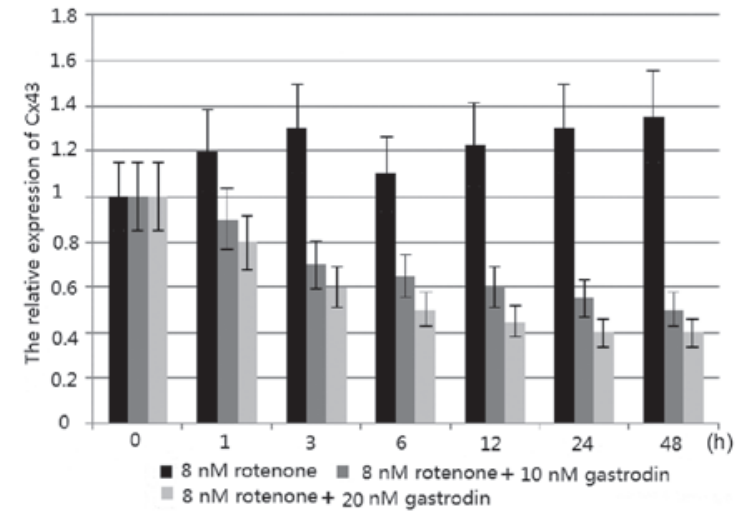

Figure 2. qPCR analysis of connexin 43 (Cx43) mRNA expression. Astrocytes were cultured with rotenone $(8 \mathrm{nM})$ and gastrodin $(10$ or $20 \mathrm{nM})$ for $0-48 \mathrm{~h}$. The relative expression of $\mathrm{Cx} 43$ was calculated as relative to the value of CX43 expression in untreated astrocytes (control). Cx43 mRNA levels were normalized by glyceraldehyde 3-phosphate dehydrogenase (GAPDH) mRNA, the level of which did not change during culture with rotenone (data not shown). Values are presented as the mean $\pm \mathrm{SE}(\mathrm{n}=3)$. Gastrodin reduces the mRNA levels of $\mathrm{Cx} 43(\mathrm{P}<0.05)$.

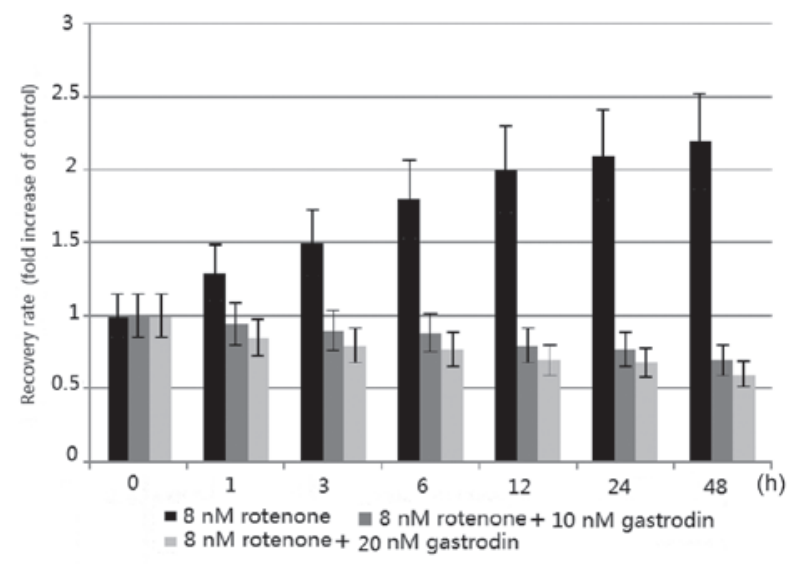

Figure 3. Dose analyses of the effect of rotenone and gastrodin on gap junctional intercellular communication (GJIC) in cultured astrocytes. GJIC was assessed by fluorescence recovery after photobleaching (FRAP), in terms of the recovery rate (RR; fold increase of control cells). Astrocytes were cultured with rotenone $(8 \mathrm{nM})$ and gastrodin $(10$ or $20 \mathrm{nM})$ for $0-48 \mathrm{~h}$. Columns show the fold increase in RR compared with that of untreated cells for $0-48 \mathrm{~h}$. Gastrodin reduce the amount of GJIC in cultured astrocytes $(\mathrm{P}<0.05)$.

of gastrodin; levels of inhibition were greatest following the addition of $20 \mathrm{nM}$ gastrodin (Fig. 2).

GJIC is upregulated by rotenone and downregulated by gastrodin. The effect of rotenone and gastrodin on GJIC in cultured astrocytes was observed. The GJIC was quantitatively assessed in living cells by a FRAP assay, as previously described (23), in terms of the RR. Following photobleaching, sequential scans detected the recovery of fluorescence in the bleached cells as the dye was transferred from the surrounding non-bleached cells to the photobleached cells through GJIC. The RR at $48 \mathrm{~h}$ of treatment showed a dose-dependent increase up to $8 \mathrm{nM}$ rotenone (23). In addition, time course analysis showed a time-dependent increase in GJIC following rotenone treatment (23). The quantity of GJIC was consistent with the expression levels of phosphory-
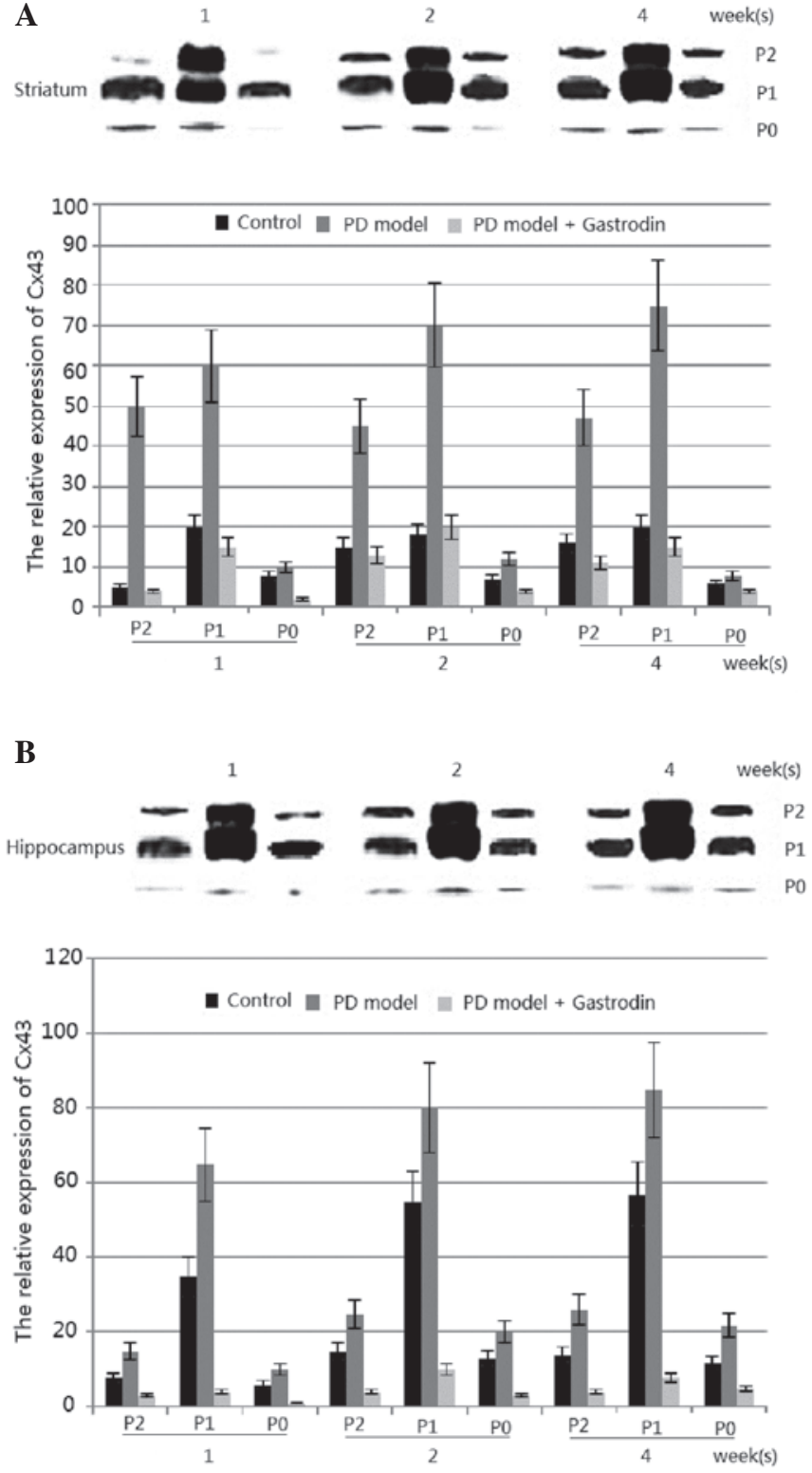

Figure 4. Cx43 protein levels in the brain. (A) $\mathrm{Cx} 43$ protein levels in the striatum of rotenone- and gastrodin-treated rats relative to that of panacet (vehicle)-treated rats (control) at 1,2 and 4 weeks. (B) Cx43 protein levels in the hippocampus of rotenone- and gastrodin-treated rats relative to that of panacet (vehicle)-treated rats (control) at 1,2 and 4 weeks. The concentrations of rotenone and gastrodin were 2.5 and $5.0 \mathrm{mg} / \mathrm{kg}$. Western blot analysis of expression levels of $\mathrm{Cx} 43$. Cx43 levels were compared between different brain regions by using identical membranes loaded with the homogenates obtained from the different regions. The graph depicts the fold increase of total $\mathrm{Cx} 43$ expression relative to the control (thalamus). $\mathrm{Cx} 43$ protein levels in the striatum and hippocampus of rotenone- and gastrodin-treated rats relative to that of panacet (vehicle)-treated rats (control) were inhibited by gastrodin $(\mathrm{P}<0.01)$. Values are mean $\pm \mathrm{SE}$ with $\mathrm{n}=3$. $\mathrm{P} 0$, non-phosphorylated form of $\mathrm{Cx} 43 ; \mathrm{P} 1 / \mathrm{P} 2$, phosphorylated forms of $\mathrm{Cx} 43$.

lated $\mathrm{Cx} 43$ induced by rotenone (Figs. 1 and 3). The results suggested that rotenone treatment of cultured astrocytes generated increased levels of phosphorylated proteins and a broadened membrane distribution of $\mathrm{Cx} 43$, which in turn led to the enhancement of GJIC.

By contrast, the concentration of gastrodin was inversely correlated with the levels of GJIC. The data from Fig. 1 suggested that gastrodin treatment of cultured astrocytes 
generated reduced levels of phosphorylated $\mathrm{Cx} 43$, and this in turn led to the reduction in GJIC (Fig. 3). Thus, gastrodin may prevent PD by inhibiting the phosphorylation of Cx43 and reducing the expression of $\mathrm{Cx} 43$.

Gastrodin and rotenone demonstrate antagonistic functions in a PD model. To investigate whether Cx43 levels may be altered in Parkinsonism, the $\mathrm{Cx} 43$ protein level in the rotenone-induced model of PD in rats was observed. In this model, Cx43 was identified in all regions (although at different levels) and the $\mathrm{Cx} 43$ protein level was significantly lower in the striatum and hippocampus than in the other brain regions (data not shown) (23). The levels of phosphorylated Cx43 were markedly enhanced in the striatum of the treated group. Significant differences in the total Cx43 levels were observed in the striatum of rotenone-treated rats at 1,2 and 4 weeks, as well as in the hippocampus of rotenone-treated rats at those weeks $(\mathrm{P}<0.01$; Fig. 4). However, no significant changes were observed in other regions (data not shown) (23). The results from Fig. 4 suggested that treatment of the rat model with gastrodin reduced the protein levels of phosphorylated $\mathrm{Cx} 43$, which was lower than that of the control group $(\mathrm{P}<0.01)$.

By contrast, the concentration of gastrodin was inversely correlated with the expression of phosphorylated $\mathrm{Cx} 43$ in the PD model induced by rotenone. The results from Fig. 4 suggested that gastrodin treatment in the rat model generated reduced protein levels of phosphorylated $\mathrm{Cx} 43(\mathrm{P}<0.01)$, which may be less than those of the control group (Fig. 4). Thus, gastrodin may be used for the prevention of PD by inhibiting the phosphorylation of $\mathrm{Cx} 43$ and reducing the expression of Cx43 in the PD model.

\section{Discussion}

Cx43 electrophoresis studies have identified three forms of Cx43, non-phosphorylated Cx43 (P0) and two slower migrating forms (commonly termed P1 and P2). The P1 and P2 isoforms were found to be associated with gap junction structures (23). In the present study, rotenone treatment induced an increase in Cx43 P1 and P2 levels in astrocytes and PD models (Figs. 1 and 4), and the number of localized foci of the total and phosphorylated $\mathrm{Cx} 43$ on the plasma membrane was increased. Furthermore, astrocyte GJIC was increased with rotenone treatment (Fig. 3). These results are consistent with those of a previous study (23). Figs. 1-4 suggest that all increases in Cx43 levels induced by rotenone were inhibited by gastrodin. Thus, gastrodin may be used for the prevention of PD, as it inhibited the phosphorylation of $\mathrm{Cx} 43$ and reduced the expression of $\mathrm{Cx} 43$ in the models. Therefore, it may be a potential therapeutic alternative for PD.

Connexins require an integrated network for protein synthesis, assembly, gating, internalization, degradation and feedback control, all of which are required to regulate the biosynthesis and turnover of gap junction channels. Fundamentally, the introduction of sequence-altering modifications results in changes in protein conformation, activity, charge, stability and localization. Thus, an understanding of the sites, patterns and magnitude of protein post-translational modification, including phosphorylation, is essential. Previously, studies of connexin phosphorylation have suggested that one or a small number of sites of modification strictly correspond to one molecular function; however, connexins undergoing multiple levels of multi-site phosphorylation are critical to improving the functions of connexin (30). The present study on rotenone-treated rats demonstrated the induction of phosphorylated $\mathrm{Cx} 43$ in astrocytes, which may be important since astrocytes exhibit direct, active and critical roles in mediating neuronal survival and function in various neurodegenerative disorders, including PD (17). The post-translational modification was inhibited by gastrodin via the suppression of Cx43 expression.

GJIC is involved in cellular growth control and may be restored by $\mathrm{Cx} 43$ protein expression; therefore, $\mathrm{Cx} 43$ is correlated with GJIC (31). The central question is whether the elevation of astrocyte GJIC is involved in the development of PD or whether it is merely a protective response to rotenone. The results of the present study demonstrated that the quantity of GJIC was correlated with the expression levels of $\mathrm{Cx} 43$. The expression levels of $\mathrm{Cx} 43$ were enhanced in the striatum and hippocampus of the PD model (Fig. 4). Subsequently, GJIC was also increased in the PD model, thus the elevation of astrocyte GJIC may result in the development of PD. Immunohistological analysis suggested that Cx43 was upregulated in astrocytes in the striatal and hippocampal regions, while the upregulation was inhibited by gastrodin. Therefore, this difference in the density of astrocytes may have affected the induction of $\mathrm{Cx} 43$ protein by gastrodin. Another possibility is that astrocytes in the striatum and hippocampus demonstrated different characteristics compared with those in other areas $(33,34)$.

Gastrodin is the main component extracted from the rhizome of Gastrodia elata (Orchidaceae), a Chinese herbal medicine, which has long been used for treating dizziness, epilepsy, stroke and dementia (35). Gastrodin exhibits a neuroprotective action against hypoxia in cultured cortical neurons, and the mechanism may involve decreasing the extracellular glutamate level (35). In the treatment of PD, gastrodin has been observed to inhibit neuroinflammation in a rotenone-induced model of PD (12). In the present study, it was demonstrated that gastrodin prevented the development of PD by downregulating the expression of $\mathrm{Cx} 43$.

In conclusion, a rat PD model was successfully set-up by treatment with rotenone. Using the rat PD model, the effects of gastrodin on PD were explored. Gastrodin can ameliorate PD by downregulating the protein levels of phosphorylated $\mathrm{Cx} 43$, which is closely correlated with the amounts of GJIC. In the rat $\mathrm{PD}$ model induced by rotenone, phosphorylated $\mathrm{Cx} 43$ was selectively enhanced in the striatum and hippocampus. The enhanced activity could be inhibited specifically by gastrodin treatment $(\mathrm{P}<0.01)$. This study also has limitations, for instance, the inhibition of Parkinsonism by gastrodin will need to be examined further in patients. It will also be necessary to examine the changes in the signal transduction of neuron cells undergoing gastrodin treatment (36). In future, gastrodin may offer a potential therapeutic alternative for PD.

\section{References}

1. Paisán-Ruíz C, Jain S, Evans EW, et al: Cloning of the gene containing mutations that cause PARK8-linked Parkinson's disease. Neuron 44: 595-600, 2004. 
2. Zhang X,LuL,Liu S, Ye W, Wu J and Zhang X: Acetylcholinesterase deficiency decreases apoptosis in dopaminergic neurons in the neurotoxin model of Parkinson's disease. Int J Biochem Cell Biol 45: 265-272, 2013.

3. Salama M, Ellaithy A, Helmy B, et al: Colchicine protects dopaminergic neurons in a rat model of Parkinson's disease. CNS Neurol Disord Drug Targets 11: 836-843, 2012.

4. Ahn EH, Kim DW, Shin MJ, et al: PEP-1-ribosomal protein $\mathrm{S} 3$ protects dopaminergic neurons in an MPTP-induced Parkinson's disease mouse model. Free Radic Biol Med 55 36-45, 2013

5. Tönges L, Frank T, Tatenhorst L, et al: Inhibition of rho kinase enhances survival of dopaminergic neurons and attenuates axonal loss in a mouse model of Parkinson's disease. Brain 135: 3355-3370, 2012.

6. Ali F, Stott SR and Barker RA: Stem cells and the treatment of Parkinson's disease. Exp Neurol: Jan 6, 2013 (Epub ahead of print).

7. Nishimura K and Takahashi J: Therapeutic application of stem cell technology toward the treatment of Parkinson's disease. Biol Pharm Bull 36: 171-175, 2013.

8. Kim H: Neuroprotective herbs for stroke therapy in traditional eastern medicine. Neurol Res 27: 287-301, 2005.

9. Pearl PL, Drillings IM and Conry JA: Herbs in epilepsy: evidence for efficacy, toxicity, and interactions. Semin Pediatr Neurol 18 203-208, 2011.

10. Schachter SC: Botanicals and herbs: a traditional approach to treating epilepsy. Neurotherapeutics 6: 415-420, 2009.

11. Manavalan A, Ramachandran U, Sundaramurthi $\mathrm{H}$, et al: Gastrodia elata Blume (tianma) mobilizes neuro-protective capacities. Int J Biochem Mol Biol 3: 219-241, 2012.

12. Li C, Chen X, Zhang N, Song Y and Mu Y: Gastrodin inhibits neuroinflammation in rotenone-induced Parkinson's disease model rats. Neural Regen Res 7: 325-331, 2012 (In Chinese).

13. Karuppagounder SS, Madathil KS, Pandey M, Haobam R, Rajamma U and Mohanakumar KP: Quercetin up-regulates mitochondrial complex-I activity to protect against programmed cell death in rotenone model of Parkinson's disease in rats. Neuroscience 236: 136-148, 2013.

14. Xiong N, Long X, Xiong J, et al: Mitochondrial complex inhibitor rotenone-induced toxicity and its potential mechanisms in Parkinson's disease models. Crit Rev Toxicol 42: 613-632, 2012

15. Drinkut A, Tereshchenko Y, Schulz JB, Bähr M and Kügler S: Efficient gene therapy for Parkinson's disease using astrocytes as hosts for localized neurotrophic factor delivery. Mol Ther 20 534-543, 2012.

16. Hauser DN and Cookson MR: Astrocytes in Parkinson's disease and DJ-1. J Neurochem 117: 357-358, 2011.

17. Rappold PM and Tieu K: Astrocytes and therapeutics for Parkinson's disease. Neurotherapeutics 7: 413-423, 2010.

18. Rouach $\mathrm{N}$ and Giaume C: Connexins and gap junctional communication in astrocytes are targets for neuroglial interaction. Prog Brain Res 132: 203-214, 2001.

19. Dermietzel R, Gao Y, Scemes E, et al: Connexin43 null mice reveal that astrocytes express multiple connexins. Brain Res Brain Res Rev 32: 45-56, 2000.

20. Nagy JI and Rash JE: Connexins and gap junctions of astrocytes and oligodendrocytes in the CNS. Brain Res Brain Res Rev 32: $29-44,2000$
21. Thompson RJ and Macvicar BA: Connexin and pannexin hemichannels of neurons and astrocytes. Channels (Austin) 2: 81-86, 2008.

22. Li X and Simard JM: Multiple connexins form gap junction channels in rat basilar artery smooth muscle cells. Circ Res 84: 1277-1284, 1999.

23. Kawasaki A, Hayashi T, Nakachi K, et al: Modulation of connexin 43 in rotenone-induced model of Parkinson's disease. Neuroscience 160: 61-68, 2009

24. Ya-qin C, Yi-fan S, Hong C, Jian-ping W and Jiao D: Effects of gastrodin on $\mathrm{Cx} 43$ expression in temporal lobe cortex and hippocampus of pentylenetetrazole-induced epileptic immature rats. Journal of Lanzhou University (Medical Sciences) 34: 9, 2008.

25. Mulcahy P, O'Doherty A, Paucard A, O'Brien T, Kirik D and Dowd E: The behavioural and neuropathological impact of intranigral AAV- $\alpha$-synuclein is exacerbated by systemic infusion of the Parkinson's disease-associated pesticide, rotenone, in rats. Behav Brain Res 243: 6-15, 2013.

26. Thakur P and Nehru B: Anti-inflammatory properties rather than anti-oxidant capability is the major mechanism of neuroprotection by sodium salicylate in a chronic rotenone model of Parkinson's disease. Neuroscience 231: 420-431, 2013.

27. Care IoLARCo, Animals UoL and Resources NIoHDoR: Guide for the care and use of laboratory animals. US Department of Health and Human Services, Public Health Service, National Insititutes of Health, 1985.

28. Wisniewska-Kruk J, Hoeben KA, Vogels IM, et al: A novel co-culture model of the blood-retinal barrier based on primary retinal endothelial cells, pericytes and astrocytes. Exp Eye Res 96: 181-190, 2012.

29. Takizawa T, Gudla PR, Guo L, Lockett S and Misteli T: Allele-specific nuclear positioning of the monoallelically expressed astrocyte marker GFAP. Genes Dev 22: 489-498, 2008

30. Kruger NJ: The Bradford method for protein quantitation. Methods Mol Biol 32: 9-15, 1994.

31. Chen VC, Gouw JW, Naus CC and Foster LJ: Connexin multi-site phosphorylation: mass spectrometry-based proteomics fills the gap. Biochim Biophys Acta 1828: 23-34, 2013.

32. Jongen WM, Fitzgerald DJ, Asamoto M, et al: Regulation of connexin 43-mediated gap junctional intercellular communication by $\mathrm{Ca}^{2+}$ in mouse epidermal cells is controlled by E-cadherin. J Cell Biol 114: 545-555, 1991.

33. Baucum AJ II, Brown AM and Colbran RJ: Differential association of postsynaptic signaling protein complexes in striatum and hippocampus. J Neurochem 124: 490-501, 2013.

34. Fidalgo C, Conejo NM, González-Pardo H and Arias JL: Functional interaction between the dorsal hippocampus and the striatum in visual discrimination learning. J Neurosci Res 90: 715-720, 2012

35. Xu X, Lu Y and Bie X: Protective effects of gastrodin on hypoxia-induced toxicity in primary cultures of rat cortical neurons. Planta Med 73: 650-654, 2007.

36. Levine AJ, Harris CR and Puzio-Kuter AM: The interfaces between signal transduction pathways: IGF-1/mTor, p53 and the Parkinson Disease pathway. Oncotarget 3: 1301-1307, 2012. 